\title{
Frailty and pain in an internal medicine ward
}

\author{
Raquel Sousa Almeida ${ }^{1}$, Maria João Pinto ${ }^{1}$, João Matos Costa ${ }^{1}$
}

\section{Fragilidade e dor numa enfermaria de medicina interna}

$\mathrm{F}_{\mathrm{d}}$ ailty is a term usually defined as a syndrome of physiological decline in late life, characterized by a state of increased vulnerability to stressors. It entails a high risk of adverse outcomes, such as functional deterioration, institutionalization, hospitalization and death. Pain is also likely to have a serious impact on older people who have ${ }^{1}$ limited ability to respond to additional stressors. ${ }^{2}$

The belief that "pain is just a part of getting older" is pervasive among patients and providers, and this notion might hinder effective treatment for the problem. ${ }^{3}$

With both conditions common among the elderly, particularly in hospitalized patients, the possibility of a correlation between frailty and pain has been raised. Elderly patients comprise a large proportion of admissions to Internal Medicine wards, therefore, both frailty and pain are frequently observed in this setting.

In this letter, we report a small study which aimed to examine the correlation between frailty and pain in elderly patients admitted to an Internal Medicine ward. Consecutive patients, aged 65 or older, admitted during a 1-month period were recruited for the purposes of the study. All patients provided their written informed consent before their inclusion.

We measured frailty using the Portuguese version of Tilburg Frailty Indicator (TFI) ${ }^{4}$ and pain with the Pain Impact Questionnaire
(PIQ-6). ${ }^{5}$ Patients less than 65 years of age or with severe intellectual impairment according to the Portable Mental Status Questionnaire, ${ }^{6}$ as well as individuals who refused to participate, were excluded.

Of the 63 elderly patients admitted, 41 were included in the study. The mean age of participants was $79 \pm 6.0$ years and $70.7 \%$ of the patients were women. A total of $58.5 \%$ patients were considered frail (TFI score $\geq 6$ ). Mean TFI total score was $6.0 \pm 2.6$ points. Mean physical domain score was $3.5 \pm 1.6$, psychological domain score $2.0 \pm 1.2$ and social domain score $0.4 \pm 0.5$ points.

Pain was reported in $80.5 \%$ of the patients, who had a mean score of $58.5 \pm 8.1$ on the PIQ-6. There was a previous ambulatory prescription of pain medication in $9.8 \%$. During admission, $56.1 \%$ had analgesic therapy (87.0\% with paracetamol, as needed). At discharge, $11.1 \%$ had an analgesic therapy prescription.

Pain and frailty were higher in women and in the group aged $>80$ years, but this was not statistically significant ( $\mathrm{p}=0.07)$.

A higher PIQ-6 score was correlated with a higher total TFI, reaching statistical significance ( $r=0.442 ; p=0.004$ - Figure 1 ).

In conclusion, higher levels of pain were correlated with higher levels of frailty. The concept of pain homeostenosis, where pain could diminish the physiological reserves needed to maintain homeostasis when faced

This study was conducted at the Hospital Distrital de Santarém, Portugal - Department of Internal Medicine.

${ }^{1} M D$. Hospital Distrital de Santarém, Portugal - Department of Internal Medicine

Raquel de Sousa Almeida. Hospital Distrital de Santarém - Serviço de Medicina III - Av. Bernardo Santareno, 2005-177 - Santarém - Portugal. E-mail: almeida. raquelsousa@gmail.com

Disclosure: The authors report no conflicts of interest.

Received September 24, 2018. Accepted in final form, November, 082018.

(cc) BY 


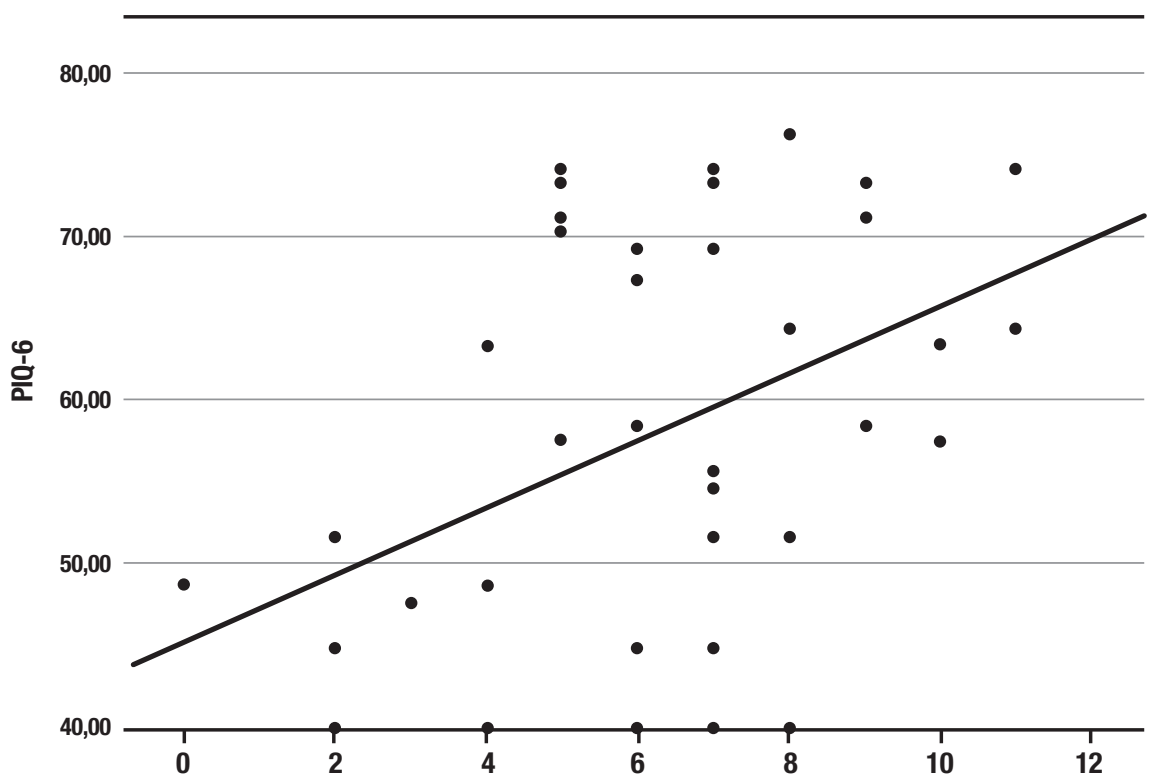

Figure 1. Correlation between Tilburg Frailty Indicator (TFI) score and Pain Impact Questionnaire (PIQ-6). with biological, psychological or social stressors, and therefore precipitate frailty, seems to be a well-supported hypothesis for this association. . $^{1,7}$

There are some limitations of this study. We recognize the multidimensional nature of frailty and pain, and that the lack of adjustment for depressive symptoms could be a bias in the study. Despite the small sample, our results emphasize the importance of early detection and interdisciplinary intervention for pain, especially in the elderly population, to help prevent vulnerability and reduce the incidence of complications. Hospitalization is an opportunity to intervene, but there is still much to be done in raising awareness of health professionals.

Authors contribution. Raquel Almeida: Wrote the paper and contributed to data collection. Maria João Pinto: contributed to data collection. João Matos Costa: Reviewed the manuscript.

Acknowledgments. We extend our thanks to the Hospital de Santarém Internal Medicine Department nursing team, especially Marta Sousa and Sónia Malaca.

\section{REFERENCES}

1. Coelho T, Paúl C, Gobbens RJ, Fernandes L. Multidimensional frailty and pain in community dwelling elderly. Pain Med. 2017;18(4): 693-701.

2. Blyth FM, Rochat S, Cumming RG, Creasey H, Handelsman DJ, Le Couteur DG, et al. Pain Frailty and Comorbidity in Older Men the CHAMP study. Pain. 2008;140:224-30.

3. Gignac MA, Davis AM, Hawker G, Wright JG, Mahomed N, Fortin PR, et al. "What do you expect? You're just getting older": a comparison of perceived osteoarthritis-related and aging-related health experiences in middle- and older-age adults. Arthritis Rheum. 2006;55:905-12.

4. Coelho T, Santos R, Paúl C, Gobbens RJ. Fernandes L. Portuguese version of the Tilburg Frailty Indicator: Transcultural adaptation and psychometric validation. Geriatr Gerontol Int. 2015;15:951-960.
5. Cavalheiro LM, Gil JA, Gonçalves RS, Pacheco MP, Ferreira PL, Fã L. Measuring the pain impact in adults with a chronic pain condition: Adaptation and validation of the Pain Impact Questionnaire (PIQ-6) to the Portuguese culture. Pain Med. 2011;12(10):1538-43.

6. Pfeiffer E. A short portable mental status questionnaire for the assessment of organic brain deficit in elderly patients. J Am Geriatr Soc. 1975; 23(10):433-41.

7. Shega JW, Dale W, Andrew M, Paice J, Rockwood K, Weiner DK. Persistent pain and frailty: A case for homeostenosis. J Am Geriatr Soc. 2012;60(1):113-7.

8. Karp JF, Shega JW, Morone NE, Weiner DK. Advances in understanding the mechanisms and management of persistent pain in older adults. $\mathrm{Br}$ J Anaesth. 2008;101(1):111-20. 